\title{
Overexpression of miR-199b-5p inhibits Ewing's sarcoma cell lines by targeting CCNL1
}

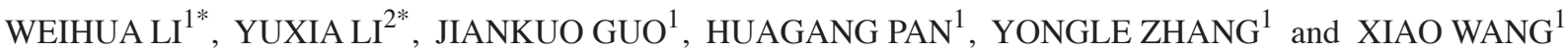 \\ ${ }^{1}$ Department of Orthopedic Surgery; ${ }^{2}$ Clinical Laboratory, \\ Henan University Hospital of Huaihe Henan University Clinical College, Kaifeng, Henan 475000, P.R. China
}

Received August 6, 2014; Accepted May 8, 2015

DOI: $10.3892 / \mathrm{mmr} .2015 .3888$

\begin{abstract}
MicroRNAs (miRNAs) are known to regulate the expression of a variety of genes, which are important in the development of several types of tumor, including Ewing's sarcoma (ES), at the post-transcriptional level. Although previous studies have identified that the expression of miRNA-199b-5p was downregulated in various types of tumor, the expression levels of miR-199b-5p in ES cells remain to be elucidated. The mechanism underlying ES via the miRNA pathway remains to be elucidated. The present study demonstrated that miR-199b-5p was an important regulator in ES cells and its expression was downregulated in ES originated A673/TC252 cells. The ES cell lines, A673 and TC252, were transfected with an miR-199b-5p mimic to overexpress the levels of this miRNA. This forced expression of miR-199b-5p suppressed the cell proliferation and invasion, arrested cell cycle progression, and promoted cell apoptosis. Furthermore, CCNL1 was identified by bioinformatic software as a potential target gene of miR-199b-5p. Following this, the present study identified CCNL1 as a direct target of miR-199b-5p in ES cells. Taken together, the present study established a functional link between ES, miR-199b-5p and CCNL1, and suggested that miR-199b-5p acts as a tumor suppressor and may be of diagnostic and therapeutic importance for human ES.
\end{abstract}

\section{Introduction}

Ewing's sarcoma (ES) is a highly malignant bone tumor occurring in children, adolescents and young adults (1), which is a type of primitive neural ectoderm family of invasive tumor (2). Patients with ES under the age of 15 comprise

Correspondence to: Professor Xiao Wang, Department of Orthopedic Surgery, Henan University Hospital of Huaihe Henan University Clinical College, 8 Baogong Lake North Road, Kaifeng, Henan 475000, P.R. China

E-mail:wangxiao0622@163.com

*Contributed equally

Key words: Ewing's sarcoma, miR-199b-5p, CCNL1 $\sim 80 \%$ of ES cases (3). Currently, clinical chemotherapy, surgery and radiotherapy, including traditional methods, are used to treat ES. However, only $60 \%$ of the patients with local disease are cured $(4,5)$. The underlying mechanism of ES remains to be elucidated.

MicroRNAs (miRNAs) are a type of endogenous non-coding small RNA molecule, 22 nucleotides in length, which regulate the expression of target genes predominantly at the post-transcriptional level $(6,7)$. Previous studies demonstrated that miRNAs were involved in almost all the human physiological activities, including cell proliferation, apoptosis, cell growth and differentiation, and provided increasing confirmation in these aspects, and at the same time also suggested that miRNAs are involved in the occurrence of various types of disease $(8,9)$. Revealing the regulatory function and mechanism of miRNAs may assist in better understanding the complex regulatory network of higher eukaryotes. This may lead to miRNAs being used clinically as convenient and practical gene therapy treatments.

miRNAs were demonstrated to be important in ES cells $(10,11)$. The present study used ES cell lines, A673 and TC252, to investigate the function and mechanism of miR-199b-5p in vitro. It was demonstrated that miR-199b-5p was a tumor suppressor in these ES cell lines, which inhibited cell proliferation and cell invasion, arrested cell cycle progression, and promoted apoptosis. In addition, it was revealed that miR-199b-5p directly targeted CCNL1 to perform this function in ES cells. Taken together, the present study successfully identified miR-199b-5p as a key regulator in ES cells.

\section{Materials and methods}

Cell lines and cell culture. The A673 and TC252 cell lines were maintained in RPMI-1640 (HyClone Corp., Logan, UT, USA), supplemented with $10 \%$ fetal bovine serum (FBS; PAA, Cölbe, Germany). The human mesenchymal stem cells (MSCs) as well as the A673 and TC252 cell lines were obtained from the American Type Culture Collection (ATCC; Rockville, MD, USA) and were cultured in Iscove's modified Eagle's medium, supplemented with 10\% FBS and platelet-derived growth factor-BB $(10 \mathrm{ng} / \mathrm{ml})$. The $293 \mathrm{~T}$ cells were obtained from ATCC and cultured in Dulbecco's modified Eagle's medium, supplemented with $10 \%$ FBS. 
Oligonucleotides and transfection. The miRNA-199b-5p mimic and scramble control molecules were obtained from Dharmacon (Chicago, IL, USA) and were transfected into the A673 and TC252 cells at a final concentration of $60 \mathrm{nM}$. The A673 and TC252 cells were mixed into complete medium following $6 \mathrm{~h}$ culture and were washed with phosphate-buffered saline (PBS) $48 \mathrm{~h}$ following transfection for the subsequent experiments.

RNA extraction and reverse transcription quantitative polymerase chain reaction $(R T-q P C R)$. The total RNA was extracted using TRIzol reagent (Invitrogen Life Technologies, Carlsbad, CA, USA), according to the manufacturer's instructions. To determine the quality and purity of the RNA, the absorption peak of RNA was detected at $260 \mathrm{~nm}$, 260/280 nm, and 260/230 nm using a NanoDrop (NanoDrop Technologies,. Inc., Thermo Fisher Scientific, Wilmington, DE, USA). The cDNA was generated using M-MLV reverse transcriptase (Invitrogen Life Technologies), according to the manufacturer's instructions.

RT-qPCR was performed using the SYBR Premix ExTaq kit (Takara Bio, Inc., Dalian, China) on the ABI PRISM 7500 real-time PCR System (Applied Biosystems, Foster City, CA, USA). All oligonucleotides were transfected into A673 and TC252 cells using Dhamafect 1 (Dharmacon). The sequences of the specific primers used for PCR were: miR-199b-5P, forward: 5'-CAGCCCAGTGTTTAGACTATC-3' and reverse: 5'-CAGTGCAGGGTCCGAGGT-3'; U6, forward: 5'-CTC GCTTCGGCAGCACATATACT-3' and reverse: 5'-ACGCTT CACGAATTTGCGTGTC-3'. The cycling conditions were as follows: $95^{\circ} \mathrm{C}$ for $3 \mathrm{~min}, 95^{\circ} \mathrm{C}$ for $5 \mathrm{sec}, 60^{\circ} \mathrm{C}$ for $30 \mathrm{sec}$, $72^{\circ} \mathrm{C}$ for $30 \mathrm{sec}$ then $72^{\circ} \mathrm{C}$ for $10 \mathrm{~min}$ for 30 cycles. The data were uniformly normalized to the internal control U6 and the relative expression levels were evaluated using the $2-\Delta \Delta \mathrm{Ct}$ method (12). The mRNA expression levels of U6 were used as endogenous control. All the experiments were performed three times.

Proliferation assay. The cells were seeded $\left(8 \times 10^{3}\right.$ cells/well) in 24-well plates and proliferation was determined using a cell counting kit-8 (CCK8; Dojindo Laboratories, Kumamoto, Japan). miR-99b-5p mimic was transfected after $16 \mathrm{~h}$. The cells were collected and digested with $0.25 \%$ trypsin, then, suspended in fresh medium. The proliferative activity was detected at $0,24,48$ and $72 \mathrm{~h}$ with CCK8. A total of $10 \mu \mathrm{l} /$ well CCK8 was added to the medium $2 \mathrm{~h}$ prior to testing at $37^{\circ} \mathrm{C}$. The absorbance in each well was measured with a microplate reader at 450 and $630 \mathrm{nM}$. (650 nm wavelength as reference wavelength; Bio-Rad Laboratories, Inc., Hercules, CA, USA).

Cell cycle analysis. The A673 and TC252 cells were cultured in serum-free medium following $4 \mathrm{~h}$ of transfection and were cultured for $24 \mathrm{~h}$, prior to the addition of complete medium. The A673 and TC252 cells were washed twice with cold PBS $48 \mathrm{~h}$ following transfection and were subsequently fixed in cold $70 \%$ alcohol overnight. The fixed cells were incubated in propidium iodide and RNase A, and were detected by flow cytometry using a C6 Flow Cytometer ${ }^{\circledR}$ Instrument (BD Biosciences, Franklin Lakes, NJ, USA).
Apoptosis assay. The A673 and TC252 cells were labeled with Annexin-V at early apoptosis and were labeled with 7AAD at late apoptosis or to indicate cell death. The collected cells were diluted to $5 \times 10^{5}$ cells $/ \mathrm{ml} 48 \mathrm{~h}$ after transfection. The cells were washed twice with cold PBS and were subsequently incubated with PE annexin-V and 7AAD (BD Biosciences, Bedford, MA, USA). The data were analysis by fluorescence-activated cell sorting.

Invasion assays. The A673 and TC252 cells were collected and adjusted at a concentration of $2 \times 10^{5}$ cells $/ \mathrm{ml} 24 \mathrm{~h}$ after transfection. Culture medium, containing $10 \%$ FBS, was added to the lower chamber (at the bottom of a 24-well plate) and the cell suspension was added to the upper chamber. The A673 and TC252 cells were maintained in culture for $24 \mathrm{~h}$. The cells in the upper chamber were washed away and stained with $0.1 \%$ crystal violet at room temperature for $15 \mathrm{~min}$. The number of cells were counted in 10 different fields under the microscope (CKX41; Olympus, Tokyo, Japan) and the total number of cells invading through the Matrigel were counted in 10 representative fields under a microscope (BD Biosciences).

Western blot analysis. The A673 and TC252 cells were collected $48 \mathrm{~h}$ after transfection, washed twice with PBS and lysed on ice in cold modified radioimmunoprecipitation buffer supplemented with protease inhibitors (Roche, Mannheim, Germany) for $30 \mathrm{~min}$. The protein concentration was detected using a Bicinchoninic Acid Protein Assay kit (Beyotime Institute of Biotechnology, Shanghai, China). An equal quantity of protein $(30 \mu \mathrm{g})$ was separated by $10 \%$ SDS-PAGE. The protein was transferred onto a nitrocellulose membrane (Millipore, Billerica, MA, USA) following electrophoresis. The membrane was blocked for $2 \mathrm{~h}$ with $5 \%$ non-fat milk and was incubated with antibodies against CCNL1 (1:200; cat. no. abc-102; Millipore), c-kit (1:100; cat. no. ab5506; Abcam, Cambridge, MA, USA) and GAPDH (1:5,000; Cell Signaling Technology, Inc., Danvers, MA, USA) overnight at $4^{\circ} \mathrm{C}$. Following incuabtion with a secondary antibody (cat. no. ZB-2301; Zhong-Shan JinQuao, Shanghai, China), detection was performed using an enhanced chemiluminescence system (Thermo Fisher Scientific, Waltham, MA, USA).

Statistical analysis. The data were analyzed by the Student's t-test (two-tailed). $\mathrm{P}<0.05$ was considered to indicate a statistically significant difference.

\section{Results}

Expression of miR-199b-5p is downregulated in ES cells. RT-qPCR revealed that the expression levels of miR-199b-5p were downregulated in the ES cell line compared with the human MSCs, as shown in Fig. 1A. The MSC bone marrow mesenchymal stem cells were used as normal scramble control ES cells. A mature miR-199b-5p mimic and scramble mimic were constructed and transfected into the cells in vitro to overexpress the levels of miR-199b-5p in the A673 and TC252 cells. The expression levels of the miR-199b-5p mimic were subsequently detected in the A673 and TC252 cells. As shown in Fig. 1B, the overexpression was considered significantly different, compared with the scramble control. Taken together, 
A

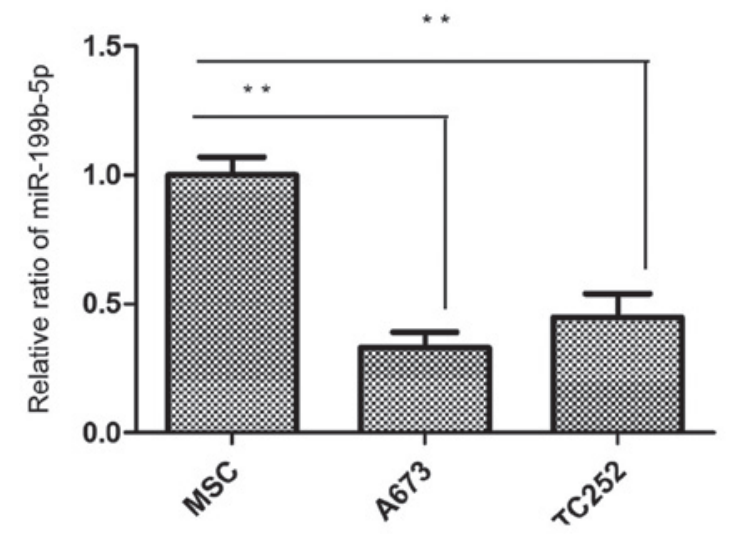

B

TC252

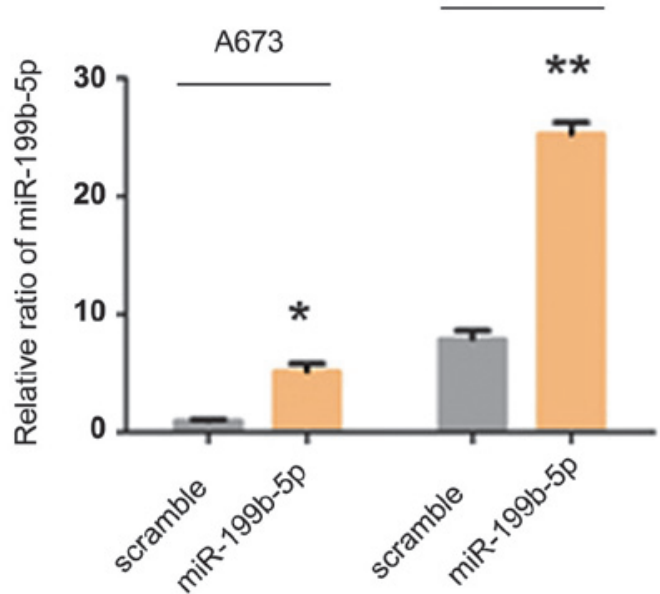

Figure 1. Expression of miR-199b-5p in ES cell lines. (A) The expression levels of miR-199b-5p in ES cell lines were significantly decreased compared with mesenchymal stem cells, as detected by reverse transcription quantitative polymerase chain reaction. (B) Forced expression of miR-199b-5p significantly upregulated the expression levels of miR-199b-5p compared with the scramble control. ( $\left(\mathrm{P}<0.05,{ }^{* *} \mathrm{P}<0.01\right.$, compared with scramble cells). ES, Ewing's sarcoma; miR, microRNA.

A

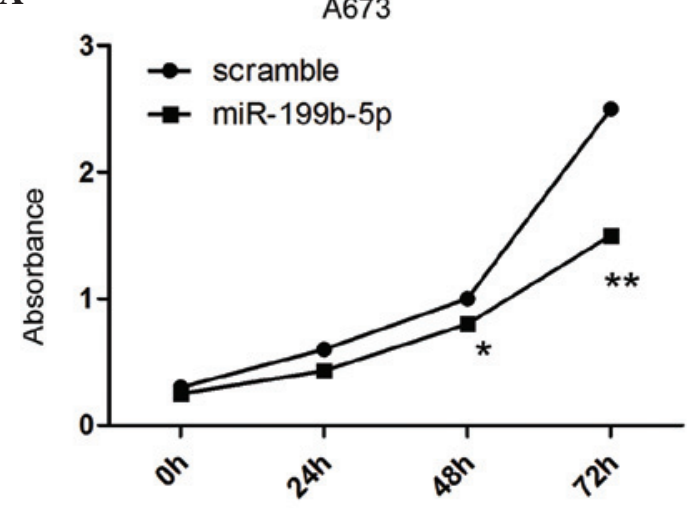

B

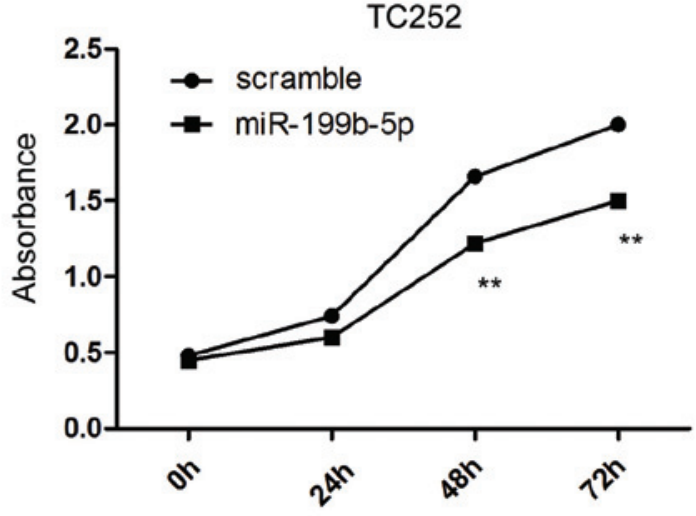

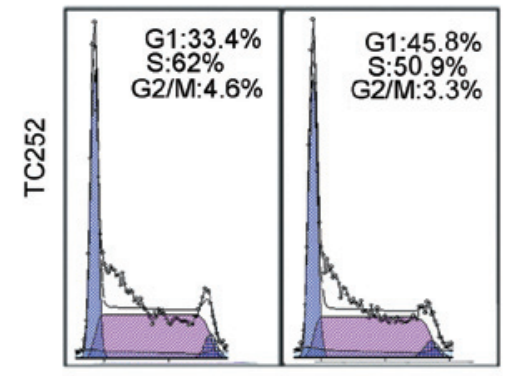
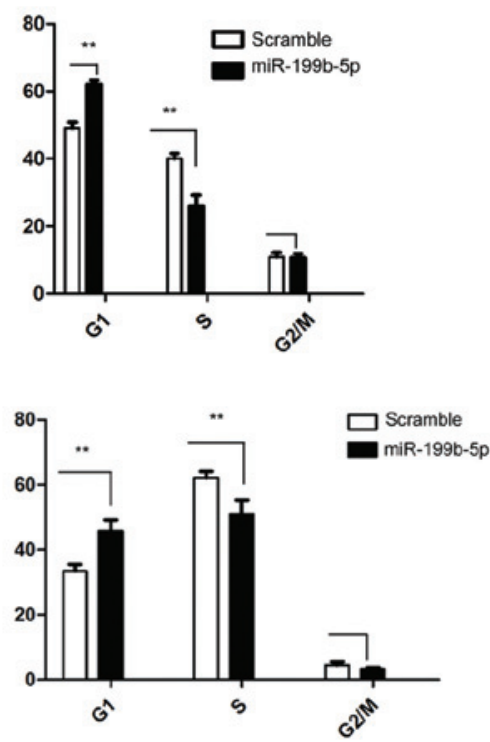

Figure 2. Ectopic expression of miR-199b-5p inhibits cell proliferation, inhibits the cell cycle at the G1- to S-phase transition, induces cell apoptosis and suppresses cell invasion in ES cells. (A) Expression of miR-199b-5p significantly inhibited the cell proliferation in A673/TC252 cells. (B) Flow cytometry was performed to assess the cell cycle and revealed that miR-199b-5p arrested cells at the G1- to S-phase transition. 

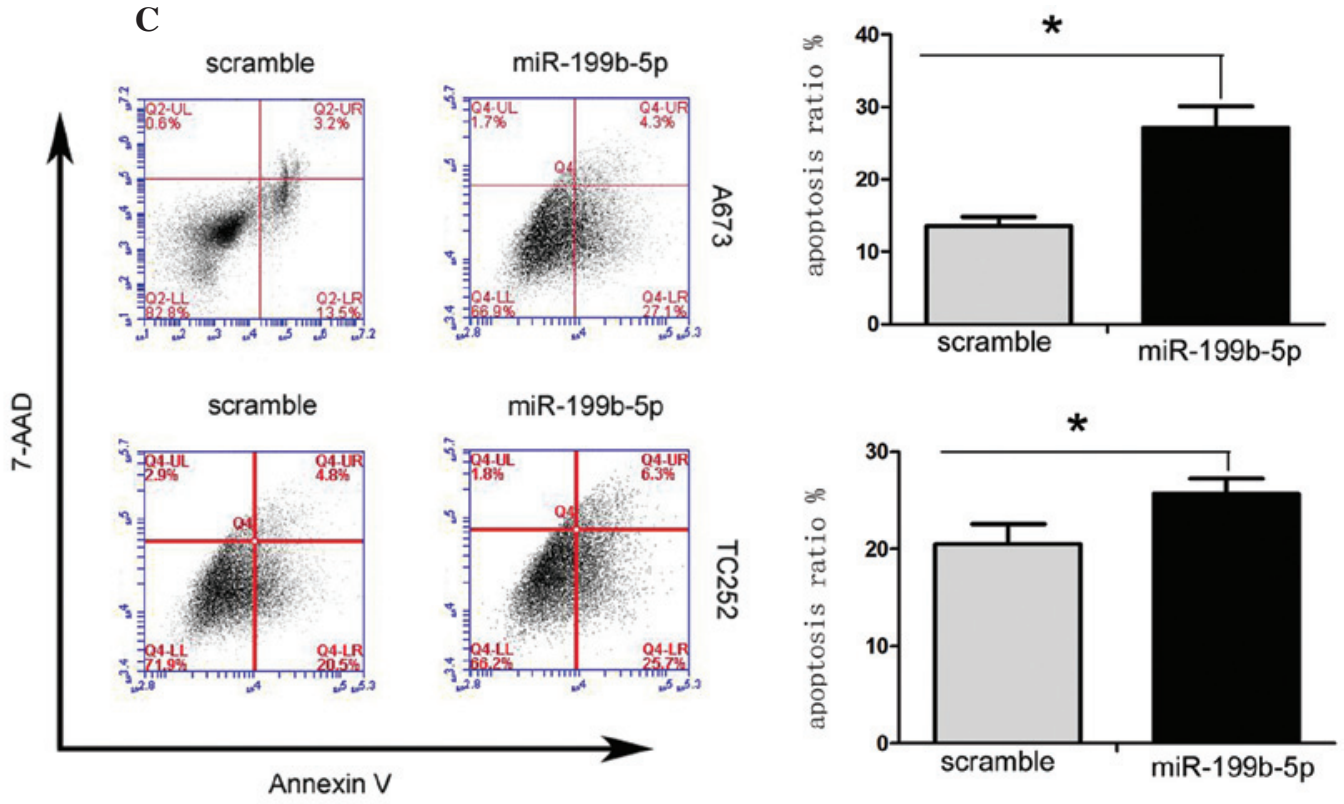

D

A673

TC252

scramble miR-199b-5p

scramble miR-199b-5p
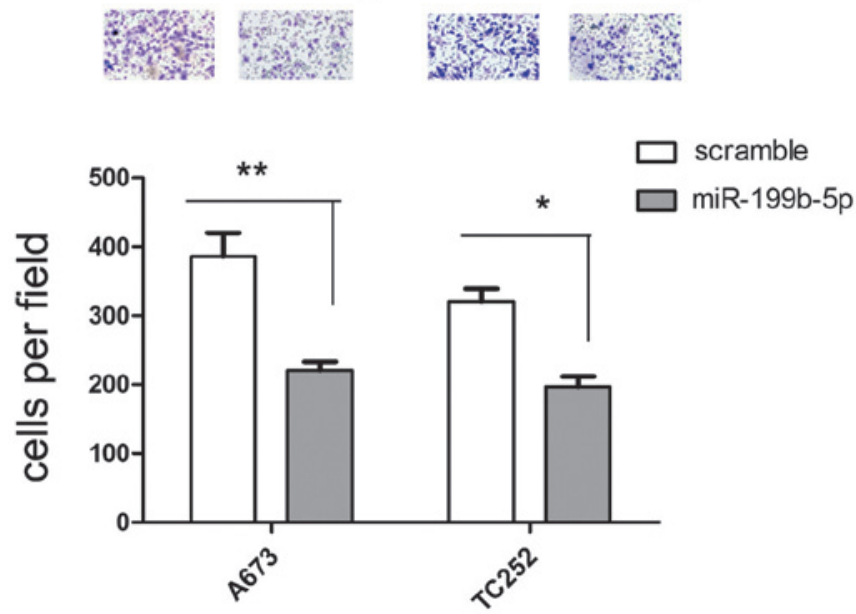

Figure 2. Continued. (C) An apoptosis assay demonstrated that miR-199b-5p induced cell apoptosis. In the dot plots, top left quadrant : dead cells; bottom left quadrant: living cells; bottom right quadrant: cells in early apoptosis; and top right quadrant: cells in late apoptosis. (D) An invasion assay revealed that miR-199b-5p suppressed the cell invasion ability. miR, microRNA.

these findings suggested that miR-199b-5p may act as a negative modulator in ES cells.

Overexpression of miR-199b-5p inhibits proliferation and invasion, inhibits cell cycle progression, and induces apoptosis in ES cells. The A673 and TC252 cells were harvested separately following transfection with miR-199b-5p mimic and scramble control at 0,24, 48 and $72 \mathrm{~h}$. The activity of A673 cells was subsequently assessed at different time points. The overexpression of miR-199b-5p significantly inhibited the cell proliferation compared with the scramble control in vitro, as shown in Fig. 2A.

Since cell proliferation was directly associated with the cell cycle, the effect of the miR-199b-5p mimic on the cell cycle was analysed. The quantity of cells in the G1 phase increased significantly following the forced expression of miR-199b-5p
(Fig. 2B). By contrast, the percentage of cells in $\mathrm{S}$ phase decreased in the A673 and TC252 cells (Fig. 2B). Therefore, the G1- to S-phase transition was inhibited by the overexpression of miR-199b-5p.

Cell apoptosis may be the cause of the change in cell growth and proliferation in the ES cells. The number of early apoptosis cells following transfection with the miR-199b-5p mimic was then assessed. The ratio of early apoptotic cells markedly increased, as detected by PE-Annexin V staining following transfection with the miR-199b-5p mimic compared with the scramble (Fig. 2C).

In addition, the cell invasive ability was determined following the forced expression of miR-199b-5p in ES cells. The Matrigel invasion chamber assays demonstrated that the invasive ability of cells with overexpression of miR-199b-5p significantly decreased (Fig. 2D). These results suggested that 

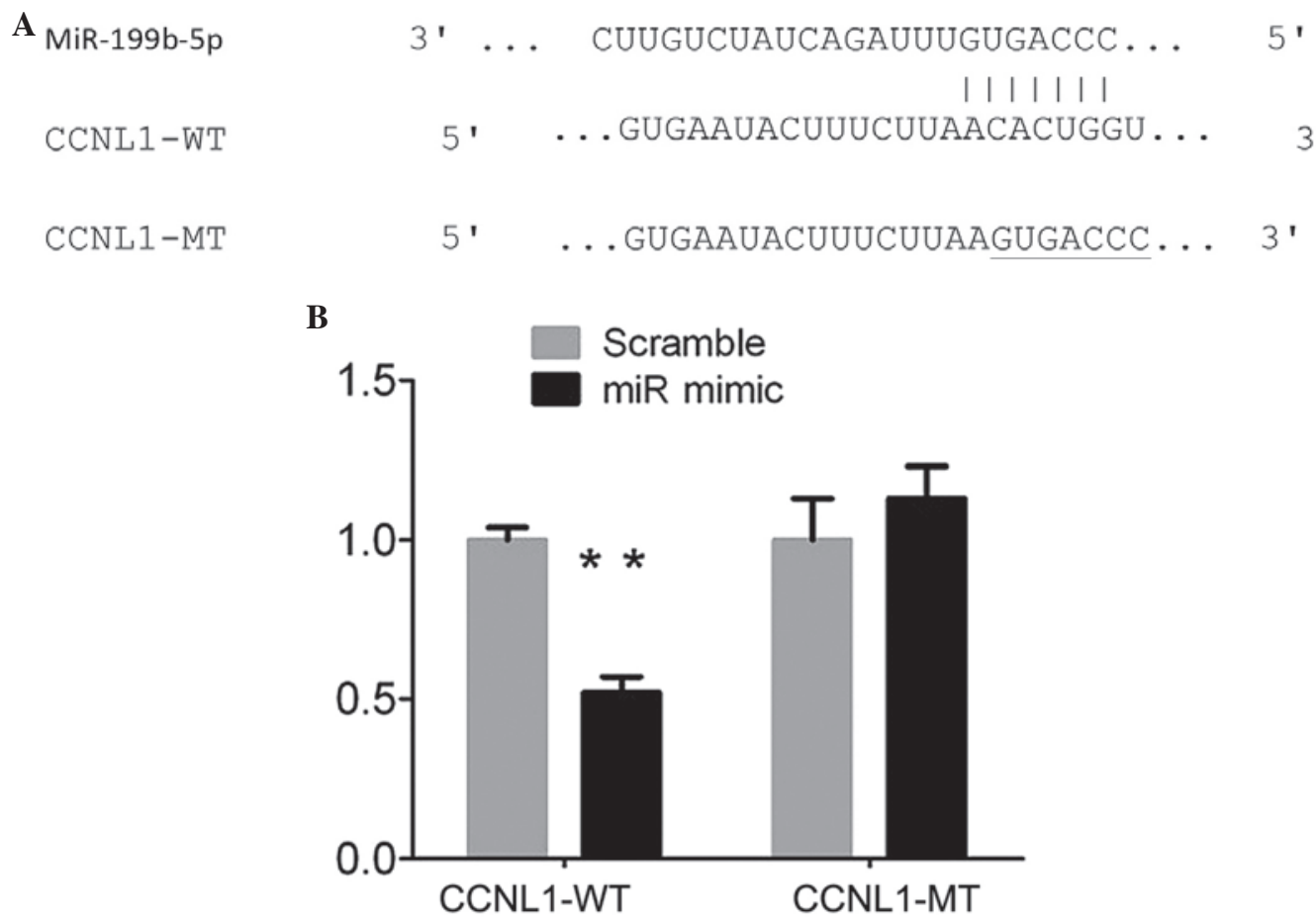

Figure 3. CCNL1 is the target gene of miR-199b-5p in vitro. (A) The nucleotide sequences of miR-199b-5p and the complementary sequence in CCNL1 mRNAs revealed the potential binding site. (B) The luciferase activity was performed in the transfected 293T cells and demonstrated a significant decrease in the mRNA expression levels of CCNL1. The miR-199b-5p mimic significantly suppressed the luciferase activity of the reporter gene, containing CCNL1-WT, however, not CCNL1-MUT. The data are expressed as the mean \pm standard deviation of three independent experiments ( $\mathrm{P}<0.05,{ }^{* *} \mathrm{P}<0.01$, compared with the scramble control). miR, microRNA; WT, wild-type; MT, mutant.

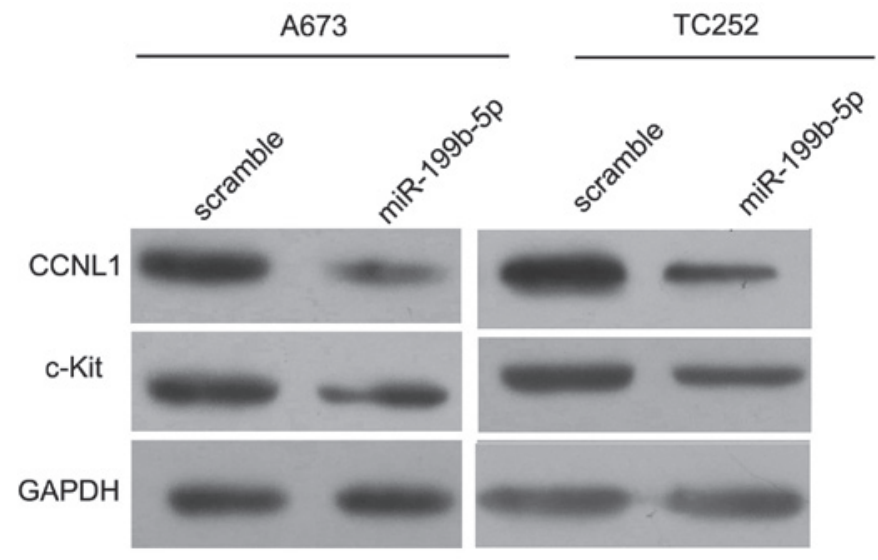

Figure 4. CCNL1 is the target gene of miR-199b, confirmed by western blotting. Western blotting demonstrated the protein expression levels of CCNL1 in the A673/TC252 cell lines following transfection with the miR-199b-5p mimic for $72 \mathrm{~h}$. miR, microRNA.

miR-199b-5p markedly inhibited cell proliferation, inhibited the cell cycle transition, induced cell apoptosis and suppressed the invasion of ES cells.

miR-199b-5p represses CCNL1 to regulate ES cells. miRNAs regulate mRNA targets by inhibiting protein translation or directly degrading the mRNA. Therefore, the present study determined potential target genes of miR-199b-5p using Targetscan (www.targetscan.org) and PicTar (www.picTar. mdc-berlin.de). CCNL1 was revealed as a possible target gene of miR-199b-5p in ES cells using a luciferase activity assay. Sequence analysis demonstrated that the 3'UTR of CCNL1 contained the miR-199b-5p binding sites (Fig. 3A). The 3'UTR of CCNL1 was cloned downstream of the pMIRreport gene, which formed CCNL1-WT and similarly, the CCNL1-MT was generated. The results revealed that the relative luciferase activity of the CCNL1-WT was significantly inhibited following transfection with the miR-199b-5p mimic compared with scramble control. However, luciferase activity of the CCNL1-MT remained unchanged (Fig. 3B). Additionally, miR-199b-5p may act as a tumor suppressor by repressing the expression of CCNL1 in ES cells.

$C C N L 1$ is the target gene of miR-199b-5p. To confirm that CCNL1 was a target gene of miR-199b-5p, the A673 and TC252 cells were collected and the total protein was extracted following the overexpression of miR-199b-5p at $72 \mathrm{~h}$. Western blotting revealed that the protein expression levels of CCNL1 markedly decreased (Fig. 4A). Therefore, miR-199b-5p may inhibit ES cells by targeting CCNL1 in vitro.

\section{Discussion}

miRNAs are important in the progression of tumor cells (13). The expression of miR-199b-5p was downregulated in a wide variety of tumor types, including ovarian cancer, breast cancer, thyroid cancer and osteosarcoma. miR-199b-5p may be downregulated by activation of the JAG1-Notch1 signaling pathways in ovarian cancer (14). miR-199b-5p has been demonstrated to inhibit cancer cell migration and colony formation in breast cancer (15) and reduces the proliferation 
of thyroid follicular cancer cells (16). Won et al (17) revealed that miR-199b-5p is involved in the Notch signaling pathway in osteosarcoma and suggested the inhibitor of miR-199b-5p may be a potential treatment strategy to prevent osteosarcoma metastasis. Garzia et al (18) demonstrated that the expression of miR-199b-5p correlated with metastasis in medulloblastoma tumor and indicated that miR-199b-5p may be combined with radiation and chemotherapy as an auxiliary treatment to improve the antitumor effect and life quality of patients. These studies provided to suggest the benefit in identifying the role of miR-199b-5p in ES cells.

The present study assessed the expression levels of miR-199b-5p in ES A673 cells. The expression of mature miR-199b-5p in the A673 cells was similar to the result in TC252 cells. In addition, in A673 and TC252 cells the expression of miR-199b-5p was downregulated compared with the levels in human MSCs, indicating that miR-199b-5p may be involved in ES. Functional experiments indicated that the forced expression of miR-199b-5p suppressed cell proliferation rate, cell invasion, arrested the cell cycle and induced cell apoptosis in each ES cell line. Bioinformatic prediction revealed CCNL1 as a predicted target gene of miR-199b-5p.

Notably, CCNL1 was demonstrated as a direct target gene of miR-199b-5p by measuring luciferase activity and protein expression levels. CCNL1, a cell cycle regulatory protein and a potential oncogene, is localized in the $3 q 25$ region and associated with the survival rate of patients with head and neck squamous cell carcinoma. For instance, CCNL1 was expressed and amplified in human head and neck squamous cell carcinoma, and was suggested as an oncogene $(19,20)$.

In conclusion, the present study has demonstrated that miR-199b-5p acted as a tumor suppressor by targeting CCNL1 in ES cell lines. These findings may provide a novel insight into the molecular mechanism underlying human ES. Furthermore, miR-199b-5p may be a novel diagnostic marker or therapeutic target for the treatment of human ES in the future.

\section{Acknowledgements}

This study was supported by the National Nature Science Foundation of China (no. 51375142) and the Ministry of Health Nature Science Foundation of China (nos. W2013ZT134 and W2013ZT135).

\section{References}

1. Windsor R, Strauss S, Seddon B, et al: Experimental therapies in Ewing's sarcoma. Expert Opin Invest Drugs 18: 143-159, 2009.

2. Teicher BA, Bagley RG, Rouleau C, et al: Characteristics of human Ewing/PNET sarcoma models. Ann Saudi Med 31: 174-182, 2011.

3. Zhang ZZ, Huang L, Yu ZM, et al: Let-7a Functions as a tumor suppressor in Ewing's sarcoma cell lines partly by targeting cyclin-dependent kinase 6. DNA Cell Biol 33: 136-147, 2014.

4. Borinstein SC, Beeler N, Block JJ, et al: A decade in banking ewing sarcoma: a report from the children's oncology group. Front Oncol 3: 57, 2013.

5. Dylla L, Moore C and Jedlicka P: MicroRNAs in Ewing sarcoma. Front Oncol 3: 65, 2013.

6. Ambros V: The functions of animal microRNAs. Nature 431: 350-355, 2004.

7. Lujambio A and Esteller M: How epigenetics can explain human metastasis: a new role for microRNAs. Cell Cycle 8: 377-382, 2009.

8. Bartel DP: MicroRNAs: target recognition and regulatory functions. Cell 136: 215-233, 2009

9. Castilla-Llorente V, Nicastro G and Ramos A: Terminal loop-mediated regulation of miRNA: selectivity and mechanisms. Biochem Soc Trans 41: 861-865, 2013.

10. Li J, You T and Jing J: MiR-125b inhibits cell biological progression of Ewing's sarcoma by suppressing the PI3K/Akt signalling pathway. Cell Prolif 47: 152-160, 2014.

11. Nugent M: MicroRNA function and dysregulation in bone tumors: the evidence to date. Cancer Manag Res 6: 15-25, 2014.

12. Liu H, Huang L, Zhang Z, et al: LIM mineralization protein-1 inhibits the malignant phenotypes of human osteosarcoma cells Int J Mol Sci 15: 7037-7048, 2014.

13. Iorio MV and Croce CM: MicroRNA dysregulation in cancer: diagnostics, monitoring and therapeutics. A comprehensive review. EMBO Mol Med 4: 143-159, 2012.

14. Liu MX, Siu MK, Liu SS, et al: Epigenetic silencing of microRNA-199b-5p is associated with acquired chemoresistance via activation of JAG1-Notch1 signaling in ovarian cancer. Oncotarget 5: 944-958, 2014.

15. Fang C, Zhao Y and Guo B: MiR-199b-5p targets HER2 in breast cancer cells. J Cell Biochem 114: 1457-1463, 2013.

16. Rossing M, Borup R, Henao R, et al: Down-regulation of microRNAs controlling tumourigenic factors in follicular thyroid carcinoma. J Mol Endocrinol 48: 11-23, 2012.

17. Won KY, Kim YW, Kim HS, Lee SK, Jung WW and Park YK: MicroRNA-199b-5p is involved in the Notch signaling pathway in osteosarcoma. Hum Pathol 44: 1648-1655, 2013.

18. Garzia L, Andolfo I, Cusanelli E, Marino N, Petrosino G, De Martino D, Esposito V, et al: MicroRNA-199b-5p impairs cancer stem cells through negative regulation of HES1 in medulloblastoma. PLoS One 4: e4998, 2009.

19. Redon R, Hussenet T, Bour G, et al: Amplicon mapping and transcriptional analysis pinpoint cyclin $\mathrm{L}$ as a candidate oncogene in head and neck cancer. Cancer Res 62: 6211-6217, 2002.

20. Sticht C, Hofele C, Flechtenmacher C, et al: Amplification of Cyclin L1 is associated with lymph node metastases in head and neck squamous cell carcinoma (HNSCC). Br J Cancer 92: 770-704, 2005. 